\title{
Diagnostic et gestion des réseaux d'assainissement pluviaux et unitaires : la mesure, outil de base de la gestion en matière de lutte contre les inondations. Diagnosis and management of sewerage and urban storm networks : the measurement as a tool for flood control management.
}

\author{
S. Charton \\ Lyonnaise des eaux, Bordeaux \\ P. Hatinguais \\ D.S.T.A.E., Communauté urbaine de Bordeaux
}

Les techniques et les technologies employées pour effectuer des mesures peuvent différer selon les objectifs visés par l'action dans laquelle elles s'insèrent :

- Diagnostic.

- Contrôle et suivi du fonctionnement.

- Gestion en temps réel.

- Conception : aménagement ou extension de réseaux.

La présente communication a pour objet de proposer, parmi les nombreux paramètres pouvant caractériser le fonctionnement d'un système d'assainissement pluvial ou unitaire, ceux qu'il est souhaitable de mesurer en fonction du but poursuivi et par suite de préciser la méthodologie de la campagne, les résultats à en attendre et les contraintes d'exploitation qui en découlent.

The measurement techniques are different according fo their operational objectives :

- diagnosis

- control of operation

- management

- conception, equipment and enlargement of networks

The present paper proposes an examples of management of an urban storm network with the choice of parameters to be controlled, the methodology of measurement campaigns, the expected results and the operational constraints.

Le réseau d'assainissement pluvial d'une grande agglomération comprend un ensemble d'installations allant de la plus simple, - le collecteur, - à la plus complexe, - la station d'évacuation de crues de grosse capacité, - très dispersées géographiquement, et pratiquement impossible à gérer sans un outil de contrôle et de commande centralisé.

Ses objectifs sont :

- la maîtrise hydraulique de l'écoulement;
- la lutte contre les inondations;

- la lutte contre la pollution.

Ils définissent :

- la nature des informations à recueillir;

- les problèmes spécifiques qui s'y attachent;

- le choix des capteurs en fonction du milieu à mesurer, du système de transmission utilisable et du support de l'information pour son traitement. 


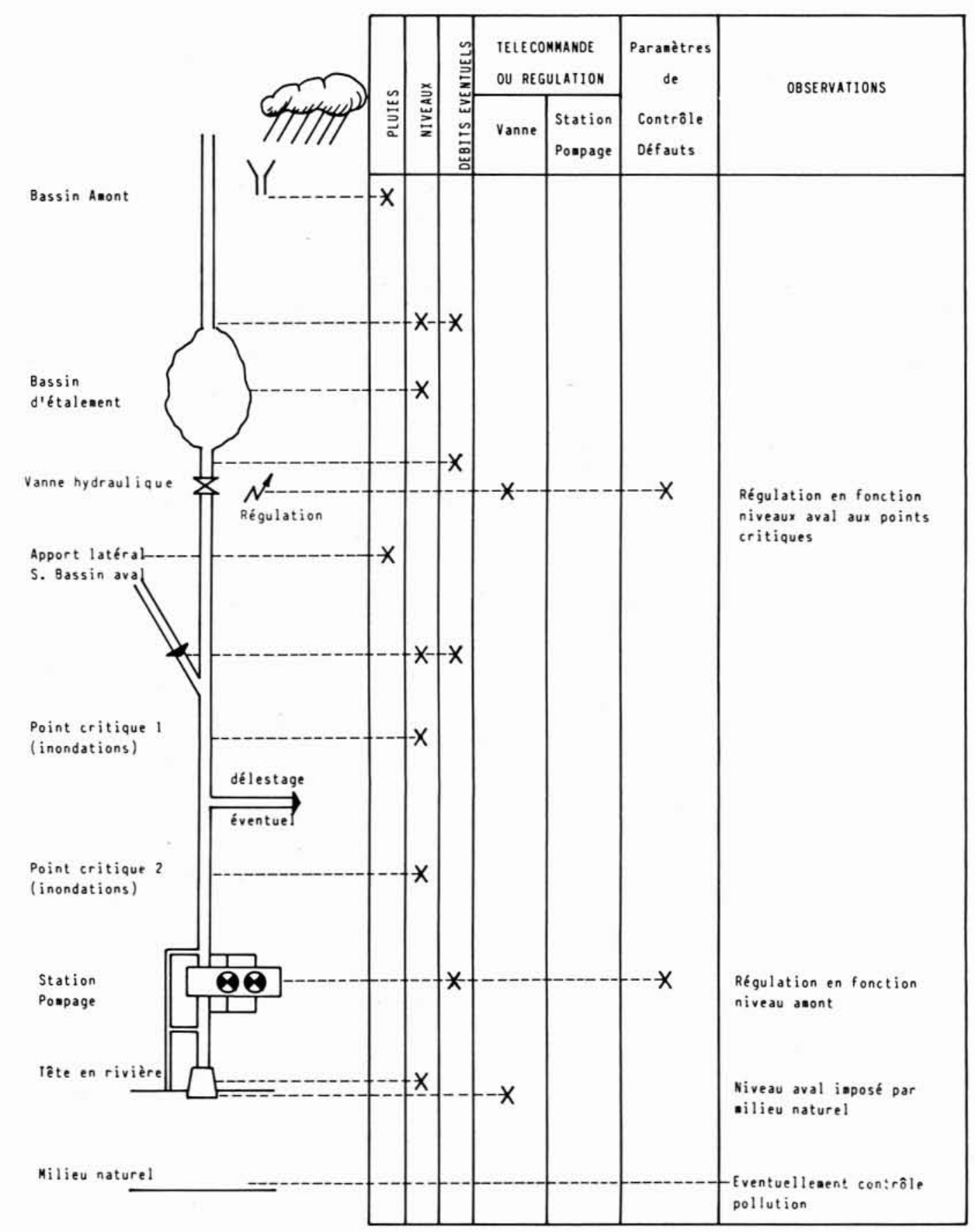

1. Maîtrise des écoulements pluviaux : paramètres à appréhender.

La figure 1 présente les différents paramètres qu'il convient de saisir.

Les quelques réflexions qui vont suivre sont tirées de l'expérience de gestion du réseau de la Communauté urbaine de Bordeaux où l'aspect "lutte contre les inondations " a, pour l'instant, un caractère prioritaire par rapport à l'aspect "régulation de la pollution".

\section{La gestion d'un réseau}

L'exploitation d'un réseau d'assainissement pluvial ou unitaire comporte deux modes de gestion totalement différents : 
- une gestion de temps sec et de petites pluies où les phénomènes aléatoires sont limités et où l'objectif principal est la maitrise des flux de pollution. Il s'y ajoute, dans le cas d'un réseau unitaire, l'optimisation du fonctionnement des stations de traitement;

- une gestion en période de pluie avec épisodes pluvieux, exceptionnels en intensité ou en durée, dite "gestion de crise ". Ces épisodes apparaissent en général de façon brutale et imprévisible; ils ne se reproduisent jamais à l'identique. Ils sollicitent simultanément et au maximum l'ensemble des installations d'un ou plusieurs bassins versants. Il est nécessaire de réagir le plus vite possible et en fonction de paramètres aléatoires, rapidement variables et interactifs.

Dans de telles situations, les principaux paramètres à prendre en compte sont :

- la pluie qui détermine l'intensité des phénomènes à maîtriser;

- les hauteurs d'eau dans les ouvrages qui traduisent les conséquences des phénomènes pluvieux;

- éventuellement les débits (à condition de savoir les mesurer), cette grandeur physique étant surtout utile en cours de régulation.

En période de crise, la rapidité d'apparition et d'évolution des phénomènes et les réactions simultanées des ouvrages nécessitent une télétransmission et une hiérarchisation des informations afin de prédisposer les actionneurs et faire de la télégestion.

\section{Les pluies}

Un des problèmes essentiels est celui du traitement de l'information fournie par les pluviographes. Le stade du simple enregistrement sur papier est dépassé; il faut évoluer vers des systèmes intégrant les données dispersées donnant une vision d'ensemble de l'épisode pluvieux et permettant de suivre son évolution. Ceci conduit à tracer les isohyètes et à calculer les lames d'eau pratiquement en temps réel.

\subsection{Les capteurs}

Les pluviographes mis en place à l'origine "Précis Mécanique type 3020 " posent un problème au niveau de l'orifice calibré qui crée des obstructions et ne permet pas d'enregistrer valablement des intensités de pluies supérieures à $1 \mathrm{~mm} / \mathrm{mn}$. De plus, le facteur temps n'est pas appréhendé avec suffisamment de précision, malgré le remplacement des horloges mécaniques par des horloges à quartz. Enfin, ces phénomènes sont sensibles aux forts écarts de température.

Ils sont progressivement remplacés par le "Précis Mécanique 3030 à transducteur " qui donne des valeurs fiables pour des précipitations allant jusqu’à $2 \mathrm{~mm} / \mathrm{mn}$. De plus, ce pluviographe délivre des impulsions qui provoquent le déplacement du stylet inscripteur du chronototalisateur, impulsions qui peuvent être traitées et télétransmises.
Enfin, nous envisageons l'essai d'un pluviographe à intensité. Le signal de sortie est traité par un microprocesseur. Un tel appareil s'affranchit des problèmes de calibrage et de transmission mécanique.

La difficulté réside dans le choix du capteur devant enregistrer la variation de hauteur d'eau: capteur à pression différentielle ou capteur à ultra-sons ?

\subsection{Cas particulier de la Communauté urbaine de Bordeaux}

Actuellement, sur les 54000 ha de superficie de la Communauté urbaine de Bordeaux, nous avons installé 32 pluviographes; cependant la partie "active " est couverte par 23 appareils, ce qui représente une densité de 1 pour 5 à $10 \mathrm{~km}$.

Ce chiffre avait été établi au départ dans l'optique des études à entreprendre sur cette zone. Les simulations ont montré que pour les besoins de la gestion du réseau en temps réel, faite au moyen de l'algorithme décrit en IV.3 (Régulation mixte par hauteur dans les collecteurs et intensité des pluies), cette densité pouvait être réduite de moitié, sans altérer les performances de la régulation.

Un contrat vient d'être passé entre le Ministère de l'Urbanisme d'une part, l'INSA de Lyon et la Lyonnaise des eaux, gestionnaire de la Communauté urbaine de Bordeaux d'autre part, pour effectuer des recherches dans le cadre du Plan urbain - sur l'influence de la densité des postes de mesures et du pas de temps retenu sur la stabilité et la continuité de la représentation spatiotemporelle de la pluie.

\subsection{Un autre outil de prévision de pluie : le radar}

La console "Météotel» constitue un outil de travail permettant de repérer, suivre, apprécier l'importance et la durée possible des précipitations dans un rayon de $200 \mathrm{~km}$ autour du centre météo. Les images sont "rafraîchies" toutes les 15 minutes.

La mise en mémoire de 15 images permet, par animation, de visualiser l'évolution des phénomènes (vitesse et direction des déplacements). Ces informations visuelles, associées aux commentaires de l'opérateur radar du centre météo, permettent la mise en état d'alerte du personnel et la possibilité d'envisager l'appel à des moyens d'intervention plus importants.

Cependant la zone d'échos fixes, couvrant une grande partie de la zone urbaine, limite l'utilisation de ces données à la prégestion des ouvrages les plus sensibles sans pour cela être assuré qu'ils seront sollicités par l'événement pluvieux.

Pour être mieux utilisé en prégestion, il sera nécessaire :

- que disparaisse la zone d'échos fixes;

- que la définition de l'image soit plus fine;

- que le temps de "rafraîchissement entre deux images ne soit pas supérieur à $5 \mathrm{~min}$;

- qu'il y ait une bonne corrélation entre pluviométrie réelle et pluviométrie imagée.

Ces études et recherches sont en cours sur ces différents points. 


\section{Les hauteurs d'eau}

La hauteur d'eau est le second paramètre de base nécessaire à un bon outil de gestion de réseau d'assainissement pluvial. Sa mesure a trois rôles principaux :

- elle permet de connaitre la charge des collecteurs aux points critiques:

- c'est un élément d'entrée dans les automatismes locaux de régulation et de pompage,

- elle entre dans le calcul des débits, soit directement couplée à un capteur de vitesse, soit indirectement à partir d'une courbe de tarage hauteur/débit.

Les capteurs: Comme pour les pluviographes, le choix d'un capteur de niveau doit être guidé par des considérations de :

- qualité de la mesure;

- temps de réponse;

- robustesse, entretien;

- alimentation en énergie (le plus souvent fonction du site);

- télétransmission;

- utilisation de l'information.

Dans des collecteurs, les contraintes de site sont importantes et les appareils doivent travailler dans des conditions difficiles dues, entre autres, au fait que les eaux véhiculent des graisses, des matières en suspension, et ce à des vitesses parfois importantes.

De ce fait, les appareils bulle à bulle sont de moins en moins utilisés, au profit des sondes piézorésistantes à pont de Wheastone qui ne nécessitent pratiquement pas d'entretien. Les seules précautions à prendre sont la protection contre les chocs et la mise à la terre des parasurtenseurs placés en amont des installations pour les protéger contre les surtensions éventuelles, leur accessibilité pour réglage et remplacement éventuels.

Le problème de l'énergie nécessaire à leur fonctionnement et de la télétransmission n'est pas négligeable. Certaines sondes admettent des résistances en ligne assez fortes entre la sonde et l'ampli, ce qui apporte une contrainte d'installation. L'alimentation sur batterie peut atteindre une autonomie de 1 mois suivant la température. Par contre, si l'on adjoint un mode de télétransmission, l'alimentation par le secteur EDF est nécessaire.

Un autre capteur de niveau présente de l'intérêt : la sonde ultrasonique, en particulier quand on l'alimente à partir de batteries solaires.

Après avoir testé plusieurs types de capteurs, pour le réseau de la Communauté urbaine de Bordeaux, nous pensons que les capteurs piézorésistifs sont ceux qui présentent actuellement le meilleur rapport qualité-prix.

De plus, on peut supporter $500 \Omega$ de résistance de ligne, ce qui permet $2000 \mathrm{~m}$ entre sonde et transducteur.

\section{Télétransmission et traitement de l'information}

Pour être à même d'utiliser avec efficacité la quantité importante de mesures recueillies à partir de ces différents capteurs, il faut pouvoir traiter ces données:
- en totalité, si c'est en temps différé (pour des besoins d'étude par exemple):

- en partie, si c'est en temps réel (dans le cas d'une gestion de crise principalement).

Deux possibilités se présentent alors :

- enregistrement local pour traitement différé sans télétransmission: sur table à dépouillement manuel, sur bande magnétique pour traitement informatique extérieur, ou sur support informatique (mémoire statique) pour traitement extérieur.

- enregistrement télétransmis sur bande papier, sur bande enregistreuse magnétique ou sur support informatique (disque dur), pour des besoins de gestion automatique locale ou de gestion centralisée.

\subsection{Télétransmission}

La télétransmission pose un problème de principe : doit-on transmettre en permanence des données qui ne sont nécessaires qu'en temps de crise ?

La transmission continue réduit l'autonomie d'un certain nombre de points de mesure. En effet, on ne dispose pas toujours d'énergie électrique sur place, et la durée des piles en batteries auxquelles on doit faire appel pour alimenter le système est limitée dans le temps.

La transmission programmée permet une plus grande autonomie. Elle fait appel par contre à un matériel de saisie doté d'une certaine intelligence qui lui permette de juger la situation en cours (comparaison de seuils par exemple). De plus, il faudra quand même faire un stockage local de façon à ne pas perdre des informations utiles pour un traitement différé.

Deux autres problèmes sont à étudier pour la télétransmission : la rapidité et la fiabilité du système.

\subsubsection{La rapidité}

Un épisode orageux est à la fois brutal et court, et ses effets sont immédiats. La décision à prendre va donc dépendre du gradient des paramètres mesurés, et ce dans un temps très court de l'ordre de quelques minutes. Ces paramètres devront donc être rafraîchis à une cadence inférieure à la minute d'où la nécessité de ne transmettre vers le P.C. que les paramètres qui varient (gradients réglables), ce qui conduit à installer des systèmes en mode déclenché au lieu du mode cyclique.

Dans le cas d'une gestion centralisée, il faut que le poste central puisse avoir une vision globale et instantanée de la situation. Ceci sous-entend une multiplicité de périphériques informatiques (imprimante, console vidéo) et conduit à un centre de gestion puissant et coûteux.

De plus, pour mieux prévoir l'imminence d'une situation de crise, il peut être intéressant d'élargir son champ de recherche en puisant des informations sur d'autres réseaux, par exemple, ceux de la Météo et du Service de la navigation.

\subsubsection{La fiabilité}

La fiabilité du système doit être une des préoccupations majeures. Les situations de crise sont, fort heureusement, de courte durée et leur nombre limité dans l'année. Mais, qu'un incident de fonctionnement survienne pendant cette 

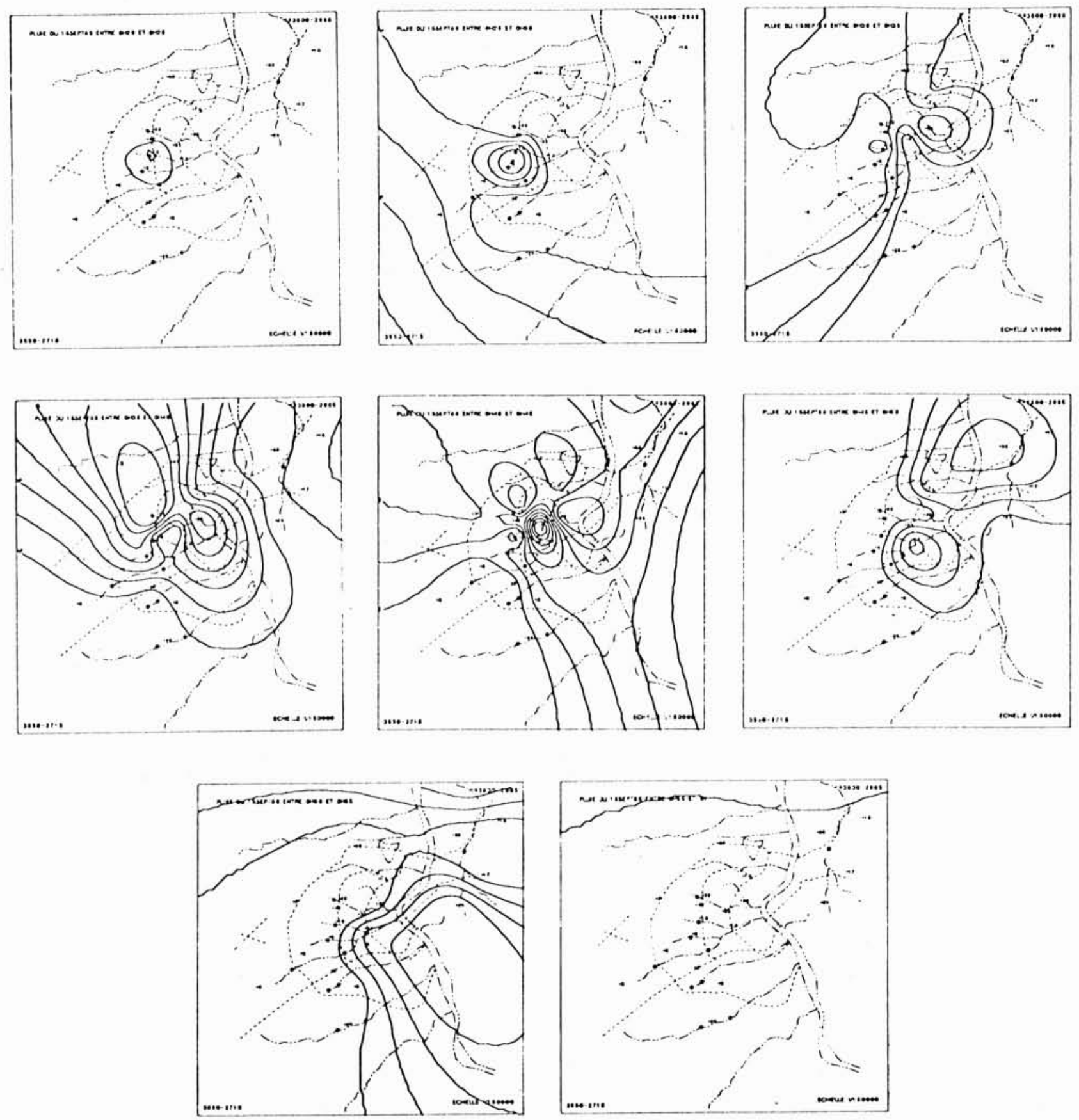

2. Suivi d'un événement pluvieux à partir des isohyètes.

période, toute la philosophie du système est remise en cause.

Sans vouloir prôner le secours absolu qui est très cher et pas toujours réalisable techniquement, il peut être profitable de s'orienter vers une structure "hiérarchisée " permettant un secours dégradé, où chaque sous-ensemble possède une certaine autonomie de fonctionnement et des enregistrements locaux.

\subsubsection{Les supports}

Les supports de transmission sont au nombre de trois : - le réseau commuté PTT, mal adapté aux conditions de rapidité et de simultanéité de l'information; - le réseau Radio, bien adapté mais souvent perturbé en période orageuse; - le réseau filaire, lignes spécialisées PTT ou lignes privées: c'est le plus utilisé. A l'étude, les fibres optiques. 

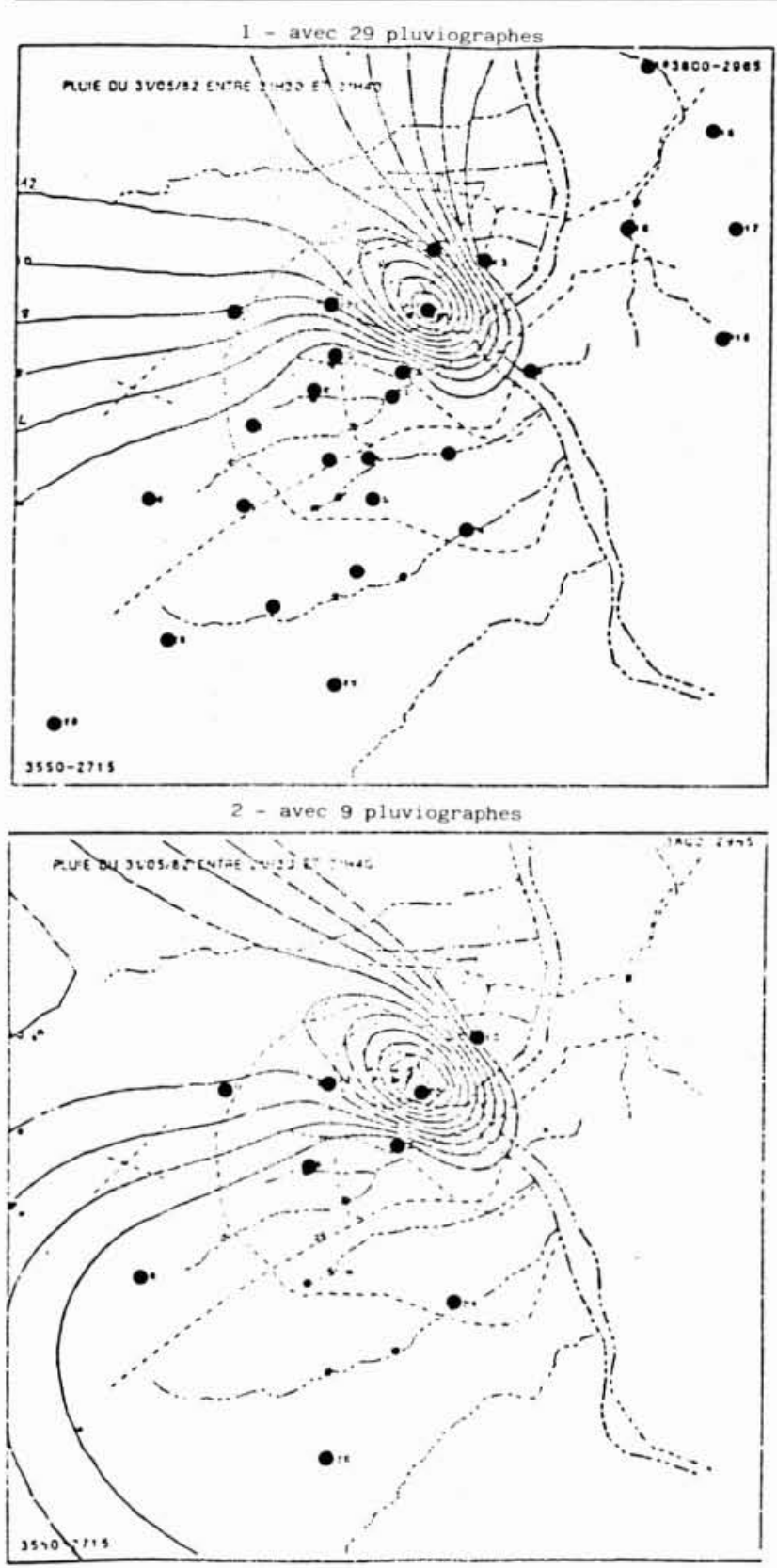

3. Tracés d'isohyètes

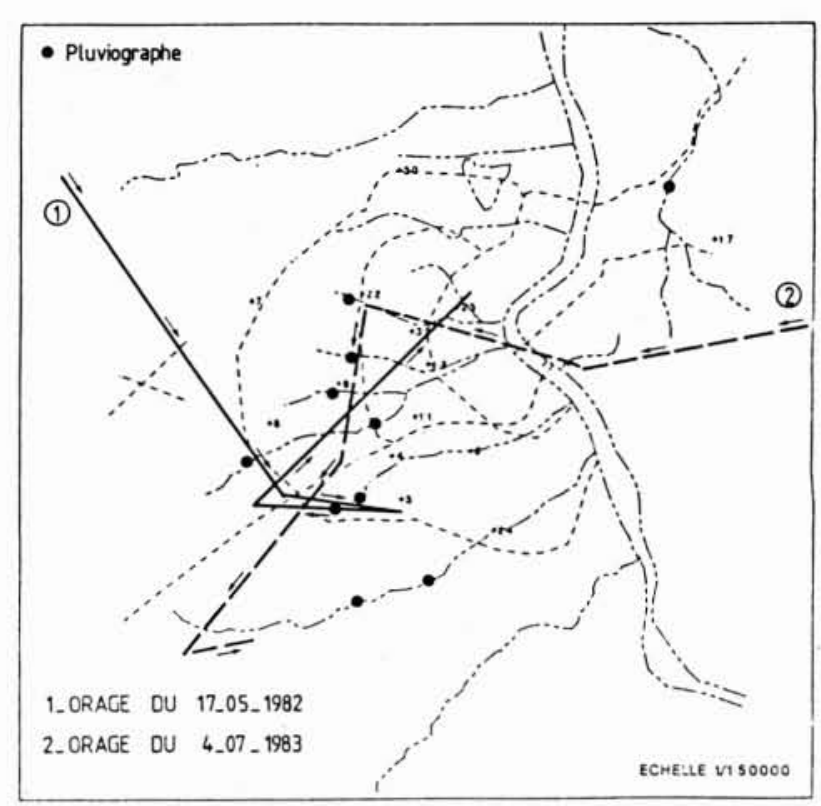

4. Suivi des points d'intensité de pluie maximale par temps de $10 \mathrm{~min}$. Peut-on parler de trajectoire?

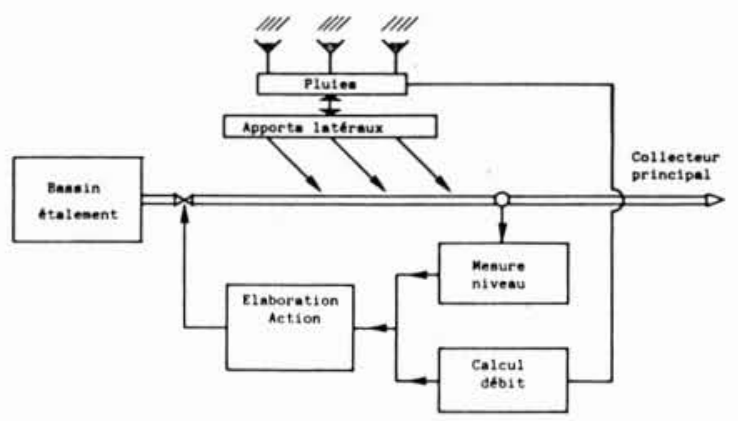

5. Schéma de régulation mixte (niveau + pluie)

\subsection{Traitement des pluies}

L'interprétation des informations données par un réseau de pluviographes ne donnera jamais qu'une image approchée de la réalité et ne permettra, en aucun cas, d'énoncer des théorèmes sur la forme des événements pluvieux.

La mesure de la pluie est par contre indispensable pour :

- la connaissance du début et de la fin de l'événement, données nécessaires à la prégestion, en particulier la mise en alerte du personnel de crise (actuellement, ce dernier est mis en alerte en fonction des indications du radar);
- l'activation des gestions locales;

- le tracé des isohyètes et le calcul des lames d'eau qui sont des paramètres d'aide à la décision.

Nous avons entrepris depuis 1983 une étude sur le tracé des isohyètes et le calcul des lames d'eau. La méthode consiste à reconstituer la pluie tombée à partir des hauteurs d'eau transmises par les pluviographes, au moyen de la fonction d'interpolation "SPLINE". Le pas de temps retenu se situe entre 5 et $10 \mathrm{~min}$ pour les orages, 15-20 min ou même plus pour les pluies de longue durée.

Les calculs sont faits à l'aide d'un micro-ordinateur HP 9836, en attendant le passage sur micro VAX 12. Le 
temps nécessaire au calcul et au tracé pour un pas de temps est de $2 \mathrm{~min} 30 \mathrm{~s}$ ce qui permet un tracé en temps réel et donc de suivre, épisode par épisode, chaque événement (fig. 2, p. 377).

En allant plus avant dans cette étude, nous avons pu formuler un certain nombre de remarques. Entre autres :

- une dizaine de pluviographes, judicieusement répartis sont suffisants pour avoir une bonne idée de l'allure des courbes isohyètes (fig. 3);

- si l'épicentre est situé en bordure de la zone d'influence des appareils retenus, la fermeture des isohyètes se fait très mal; de plus, la pluie est surrestimée si l'épicentre se trouve à proximité de l'un d'entre eux; le choix des pluviographes est donc très important;

- le déplacement de l'épicentre montre qu'il évolue de façon tout-à-fait aléatoire et qu'il paraît difficile de parler de la trajectoire d'un orage (fig. 4).

Quant à l'évaluation des lames d'eau, nous avons constaté que les valeurs obtenues dépendaient fortement du nombre de pluviographes pris en compte (écart de 30 à $40 \%$ entre 10 et 30 pluviographes).

Cet écart est évidemment intolérable pour dimensionner un projet d'aménagement de réseau.

Mais les essais de simulation montrent qu'on peut le tolérer pour une gestion "temps réel du réseau ", à condition que cette gestion soit pilotée par un algortithme de régulation en boucle mixte (en fonction des hauteurs d'eau constatées dans les collecteurs avec ajustement des consignes suivant les valeurs de la pluie) qui permet de rattraper, à l'instant " $t$ " et en dynamique, les erreurs d'estimation qui ont pu être faites à l'instant " $t-1$ ".

\subsection{Traitement des hauteurs d'eau}

Les mesures de hauteurs d'eau peuvent être traitées en temps différé ou en temps réel.

Le traitement en temps différé a un triple but dans le cadre de la gestion de réseau.

- améliorer en les précisant les consignes de fonctionnement des intercepteurs et des actionneurs;

- mieux cerner les besoins en informations (nombre de capteurs, emplacements);

- élaboration puis perfectionnement d'un système d'aide à la décision.

Le traitement en temps réel servira à deux fins :

- le fonctionnement des automatismes locaux qui régissent soit la marche des stations de pompage, soit l'utilisation des ouvrages de régulation.

Ce dernier point est particulièrement important, surtout lorsque le réseau comporte un certain nombre de bassins d'étalement. Le principe de base de la gestion est de régler le débit de fuite du bassin en fonction de la capacité maximale de transit du collecteur aval en son point le plus contraignant, tout en tenant compte des apports latéraux aval.

Une étude assez complète a conduit à trois modes de gestion possibles : gestion par les débits calculés à partir de la pluie uniquement (régulation en boucle ouverte), gestion par les hauteurs mesurées (régulation en boucle fermée), gestion mixte (boucle ouverte + boucle fermée) qui réunit les avantages des deux précédentes et peut être étendue à des cas complexes tels que bassin en série ou en parallèle (fig. 5).

- la deuxième utilisation du traitement des hauteurs d'eau en temps réel est le télétraitement au poste central. Il va permettre de modifier si nécessaire les consignes des automatismes locaux, d'agir sur des actionneurs non automatisés localement, d'utiliser des modèles d'aide à la décision lorsque l'importance du réseau l'impose. Ces modèles sont de deux types :

- catalogue de situations types, basé sur la comparaison de la situation actuelle avec toute une série de situations pré-mémorisées;

- modèle de calcul basé sur des équations simplifiées d'écoulement. Un tel modèle répond assez bien mais a besoin d'être recalé au fur et à mesure en fonction des valeurs réellement mesurées, ce qui nécessite un système bouclé.

\section{Conclusion}

Quelles qu'en soient les origines, les mesures reçues ou stockées localement doivent être l'image reelle de l'état physique constaté sur le terrain, d'où les contraintes suivantes :

- excellente fiabilité de l'installation globale;

- rapidité du suivi du phénomène physique par le capteur;

_ vitesse d'acquisition et transmission rapide (1 200 bds); - transmission vers le télécontrôle par ligne de mesure, par exemple bassin versant par bassin versant, de l'amont vers l'aval pour chacun d'eux.

Dès que le réseau a une certaine complexité, un nombre important de capteurs et de données sont nécessaires pour pouvoir appréhender le fonctionnement du réseau et le gérer au mieux (fig. 6, p. suiv.).

L'exploitation de ces données, même si ces dernières sont bien structurées et hiérarchisées, notamment en temps réel, dépasse souvent les facultés d'intégration d'un homme seul (gestion manuelle), d'où la nécessité de faire appel à l'informatique comme moyen de stockage, de traitement de l'information, et de support d'outil d'aide à la décision.

\section{Discussion} Prí-ident: M. P. HERVIO

M. HERVIO, Président de séance, indique qu'il retrouve dans l'exposé de M. Charton une démarche méthodologique et technique très proche dans ses principes de celle suivie par le Département de Seine St-Denis. La mise en place d'une gestion automatisée n'est pas une fin en soi. Il faut faire une analyse des gains en fonction du coût. Pour la Seine Saint-Denis, le coût de la gestion automatisée équivaut à $2 \mathrm{~km}$ de collecteur $\varnothing 2,50 \mathrm{~m}$ posés en agglomération. En outre on sait que les méthodes classiques d'assainissement ne permettent pas de résoudre de manière satisfaisante certains problèmes. Le développement de la gestion automatisée conduit à mettre en place un service de télésurveil- 


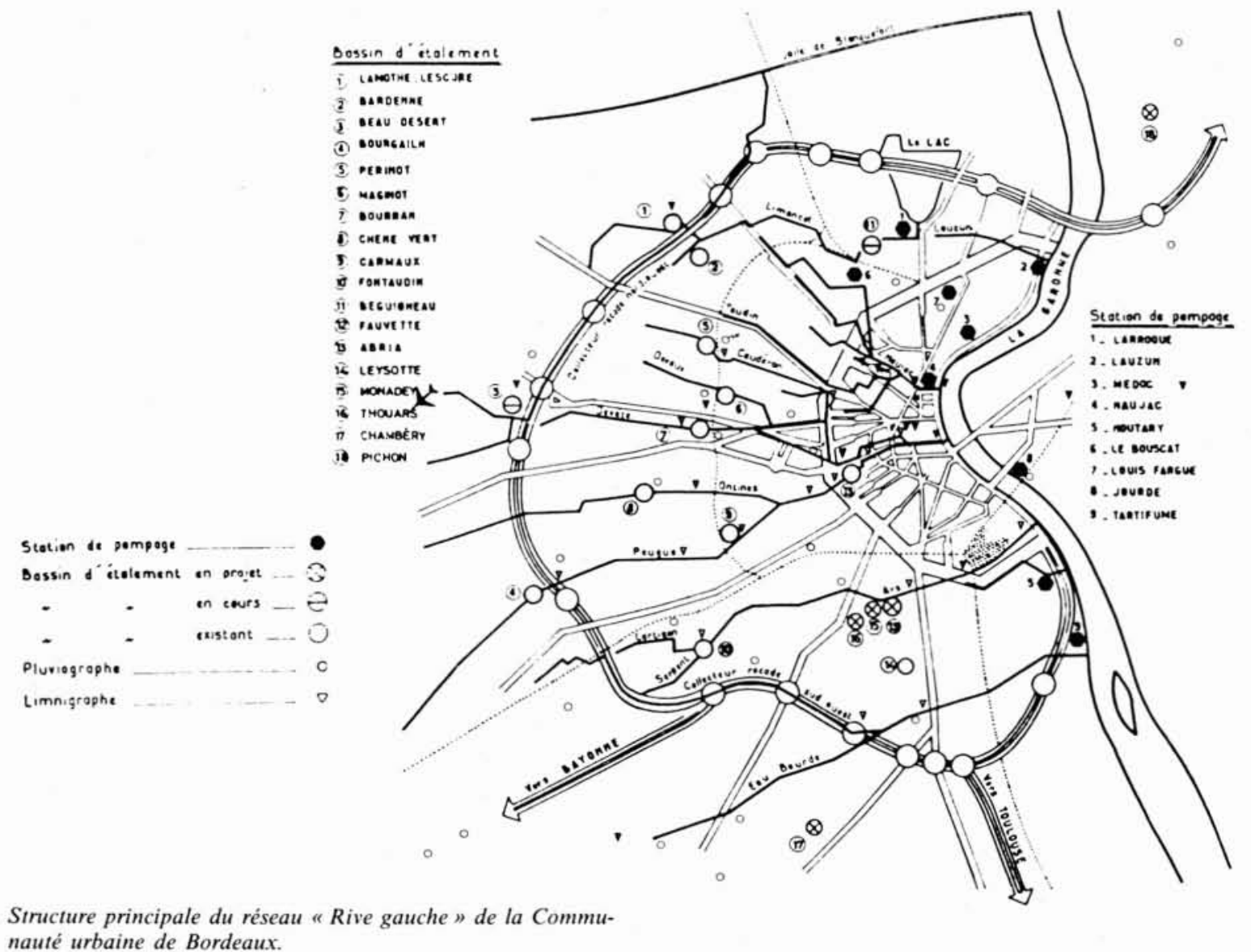

lance qui apporte une amélioration importante dans le domaine de la sécurité en égout grâce à la possibilité de dialogue entre le central et l'égoutier. On peut également assurer une alerte météorologique à l'approche d'un événement pluvieux. Après ces remarques et précisions apportées par M. HERVIO, les participants posent plusieurs questions: M. CAZENAVE demande comment techniquement sont tracées les courbes isohyètes établies par le calculateur HP. M. CHARTON indique que la table traçante est associée au calculateur.

A une question de M. ThirRiot sur la durée moyenne de fonctionnement des pompes, M. BERTRAND répond que pour les plus grosses il n'est que de quelques heures. Les stations comportent plusieurs pompes de capacités différentes, équipées de variations de vitesse et qui en période de pluie fonctionnent sur des groupes électrogènes. Par combinaison des différentes pompes on a le débit souhaité. Les grosses pompes n'interviennent que sur les pointes de débit.

La discussion s'oriente ensuite sur la densité de pluviographes nécessaire pour obtenir des courbes isohyètes précises. En réponse à une question de M. LEVY, M. CHARTON indique qu'actuellement tous les pluviographes ne sont pas télétransmis et que les courbes sont donc établies en temps différé. M. Hervio, Président de séance, fait remarquer que certains propos dans le document semblent contradictoires pour ce qui concerne la densité des pluviographes nécessaire à l'obtention d'isohyètes précises.

En fait pour la conception des réseaux, les informations doivent être précises, par contre Messieurs CHERTON et BERTRAND indiquent que pour la gestion en temps réel on peut se contenter d'un nombre de pluviographes plus restreint car ce qui compte alors c'est de réduire le temps de calcul et de bien savoir oủ se trouve l'épicentre de la pluie : on peut alors prégérer ou gérer.
Madame RouSSEL désire des précisions sur le pas de temps de la télétransmission et fait remarquer qu'elle a été frappée par la stabilité des épicentres pluvieux des événements présentés : dans ce cas est-il possible de parler de trajectoire d'orage ?

M. CHARTON répond que chaque basculement d'auget des pluviographes est traité par un automate. Le télécontrôle balaye 2000 informations par minute. Pour les pluviographes, la C.U.B. voudrait traiter une information de hauteur d'eau et non plus en fonction d'un pas de temps. M. CHARTON confirme qu'effectivement pour les deux exemples présentés, les épicentres pluvieux sont d'une remarquable stabilité. Pour ces deux épisodes pluvieux il est effectivement difficile de parler de trajectoire. M. BERTRAND précise qu'actuellement la télétransmission est basée sur l'oscultation cyclique. Bientôt la C.U.B. travaillera avec un système " maitre esclave " qui permettra de gagner du temps. A la C.U.B. les techniciens travaillent beaucoup la redondance : deux automates locaux dialoguent de manière permanente ce qui évite des pertes d'information en cas de panne de l'un d'entre eux.

M. HERVIO fait part des recherches menées par la Seine Saint-Denis et le Val de Marne dans le domaine de la prévision de la pluie à l'aide de radars météorologiques, recherches subventionnées par l'Agence de Bassin et le Plan Urbain. Un nouveau radar est installé à Trappes aux seules fins de la prévision météorologique. Cette implantation permettra d'éliminer les échos fixes et le temps de réponse sera très court (une information toutes les une ou deux minutes). Une recherche est menée en collaboration avec le CERGRENE pour corréler les images radar à l'information fournie par les pluviographes. On attend de grands progrès dans ce domaine à court terme. M. CHARTON précise que la C.U.B. a entrepris une recherche tout à fait identique, avec les mêmes objectifs que ceux décrits ci-avant. 